\title{
Association mapping of tuber eye depth and golden cyst nematode resistance traits using ICG collection of Solanum tuberosum L.
}

Totsky I.V. ${ }^{1 *}$, Rozanova I.V. ${ }^{1,2}$, Khlestkina E.K. ${ }^{1,2}$, Kochetov A.V. ${ }^{1,3}$, Safonova A.D. ${ }^{1}$

${ }^{1}$ Institute of Cytology and Genetics, SB RAS, Novosibirsk, Russia

${ }^{2}$ N.I. Vavilov All-Russian Institute of Plant Genetic Resources (VIR), St. Petersburg, Russia

${ }^{3}$ Novosibirsk State University, Novosibirsk, Russia

*e-mail: totsky@bionet.nsc.ru

Potato is one of the most important crops in the world. It's yield and quality depend on many genetic factors. The genomic regions associated with tuber eye depth and the resistance to the golden potato cyst nematode (GPCN) were found in this study by analyzing Solanum tuberosum potato varieties from the ICG "GenAgro" collection, genotyped using an Illumina 22K SNP potato array DNA chip. Data processing was carried out using programs: Tassel 5, package R, Microsoft Excel. Four statistical models were used for data analysis: GLM (general linear model) without or with $(\mathrm{GLM}+\mathrm{Q}$, $\mathrm{GLM}+\mathrm{PCA}, \mathrm{GLM}+\mathrm{Q}+\mathrm{PCA})$ taking into account the population structure. Data on resistance to the GPCN was taken from the database of the State Register of breeding achievements approved for use. The study showed the presence of 18 significant SNPs on chromosomes 1, 5 and 11. Association with three SNPs was highly significant when using each of the four statistical models. The tuber eye depth was determined on a threepoint scale: 1 - small (less than 1.0-1.3 mm), 2-medium (1.4-1.6 mm), 3-deep (more than $1.7 \mathrm{~mm}$ ). 27 significant SNPs associated with the tuber eye depth were found on chromosomes 4, 5, 6 and 10. Association with five SNPs was highly significant when using each of the four statistical models.

Acknowledgements: The Russian Science Foundation, grant number 16-16-04073, supported this work. 